\title{
Characterization of Ramp-type $\mathrm{YBa}_{2} \mathrm{Cu}_{3} \mathrm{O}_{7}$ Junctions by AFM
}

\author{
Dave H.A. Blank, Guus J.H.M. Rijnders, Roger M.H. Bergs, Martin A.J. Verhoeven, and Horst Rogalla \\ University of Twente, Applied Physics, Low Temperature Division, P.O.Box 217, 7500 AE Enschede, The Netherlands
}

\begin{abstract}
We studied the morphology of ramps in $\mathrm{REBa}_{2} \mathrm{Cu}_{3} \mathrm{O}_{7}$ (REBCO) epitaxial films on $\mathrm{SrTiO}_{3}$ substrates, fabricated by $\mathrm{RF}$ magnetron sputter deposition and pulsed laser deposition (PLD), by Atomic Force Microscopy (AFM) and High Resolution Electron Microscopy (HREM). The ramps were fabricated by $\mathrm{Ar}$ ion beam etching using different masks of standard photoresist and TiN. AFM-studies on ramps in sputter deposited films show a strong dependence, i.e., formation of facets and ridges, on the angle of incidence of the ion beam with respect to the substrate surface as well as the rotation angle with respect to the crystal axes of the substrate. Ramps in pulsed laser deposited films did not show this dependence. Furthermore, we studied the effect of an anneal step prior to the deposition of barrier layers (i.e., $\mathrm{PrBa}_{2} \mathrm{Cu}_{3-\mathrm{x}} \mathrm{Ga}_{\mathrm{x}} \mathrm{O}_{7}$ ) on the ramp. First results show a crystallization of the ramp surface, resulting in terraces and a non-homogeneous growth of the barrier material on top of it. The thickness variations, for thin layers of barrier material, can even become much larger than expected from the amount of deposited material and are dependent on the deposition and anneal conditions. HREM studies show a welldefined interface between barrier layer and electrodes. The angle of the ramp depends on the etch rate of the mask and REBCO and on the angle of incidence of the ion beam. Hard masks, like TiN, have a much lower etch rate compared to photoresist, resulting in an angle of the ramp comparable to the angle of incidence and, subsequently, in a low etching rate on the ramp.
\end{abstract}

\section{INTRODUCTION}

Nowadays, ramp-type Josephson junctions are frequently used in various applications, by the merit of their artificial barrier. In this type of junctions the barrier material and thickness can be used to tune the junction performance [1]. Mostly, Josephson junctions with a ramp-type geometry are prepared by etching a ramped edge in a multilayer of the REBCO base electrode and an isolation layer. A barrier layer and a top electrode are epitaxially grown over this structured ramp. Because, the coherence length $\xi$ is extremely short in high $\mathrm{T}_{\mathrm{c}}$ materials, barrier layers with a thickness of only a few numbers of unit cells can be used to obtain a significant dcJosephson current. Hence, surface morphology and growth of barrier material on the ramp will influence the junction properties seriously.

The product of the critical current with the normal state resistance $\left(I_{c} R_{n}\right)$, which is of the utmost importance using Josephson junctions in complex electronic devices, has a systematically higher value for lower $\operatorname{PrBa}_{2} \mathrm{Cu}_{3} \mathrm{O}_{7}$ thickness' [2]. The junction properties, however, become irreproducible and the current-voltage characteristics show large excess current when the barrier thickness is decreased below $10 \mathrm{~nm}$.

Manuscript received August 27, 1996
In this contribution we study the surface morphology of the etched ramp fabricated using a soft (photoresist) mask or hard (TiN) mask. Special attention is been paid to the effective etching of the ramped surface and the crystallization at deposition temperatures.

\section{EXPERIMENTAL}

Epitaxial $\mathrm{REBa}_{2} \mathrm{Cu}_{3} \mathrm{O}_{7}(\mathrm{RE}=\mathrm{Y}, \mathrm{Dy}, \mathrm{Pr})$ films were deposited on $5 \times 10 \mathrm{~mm}^{2}$ (001) $\mathrm{SrTiO}_{3}$ substrates using offaxis RF-magnetron sputter deposition and pulsed laser deposition, both from stoichiometric targets. Before etching the ramp, a mask of photoresist was made by spinning Shipley S-1813 for $30 \mathrm{~s}$ at $4000 \mathrm{rpm}$, and baked on a hot plate at $90^{\circ} \mathrm{C}$ for $10 \mathrm{~min}$. Thickness of the mask was about 1.2 $\mu \mathrm{m}$. Next, the sample was exposed for $15 \mathrm{~s}$ and developed. For the second approach, TiN was used as a hard mask. In this study a $40 \mathrm{~nm}$ thick TiN layer was deposited by bias sputter deposition from a Ti target (diameter of $20 \mathrm{~cm}$ ) in an $\mathrm{Ar} / \mathrm{N}_{2}$ atmosphere. Partial pressures were $2.0 \times 10^{-2}$ mbar for Ar and $0.5 \times 10^{-2}$ mbar for $\mathrm{N}_{2}$ (rf-power $=300 \mathrm{~W}$, self bias $=$ $650 \mathrm{~V}$ and substrate bias $=100 \mathrm{~V}$ ). The etching of the ramps was done with a Kaufman $\mathrm{Ar}$ ion gun $(3 \mathrm{~cm}$ in diameter, $\mathrm{V}=500 \mathrm{~V}, \mathrm{I}=10 \mathrm{~mA}$ ) at an Ar pressure of $2 \times 10^{-3}$ mbar in a pulsed mode ( $8 \mathrm{~s}$ on, $10 \mathrm{~s}$ off) to prevent overheating of the sample. The geometry during the etching process is sketched in figure 1 . The plane of incidence is perpendicular to the edge of the mask.

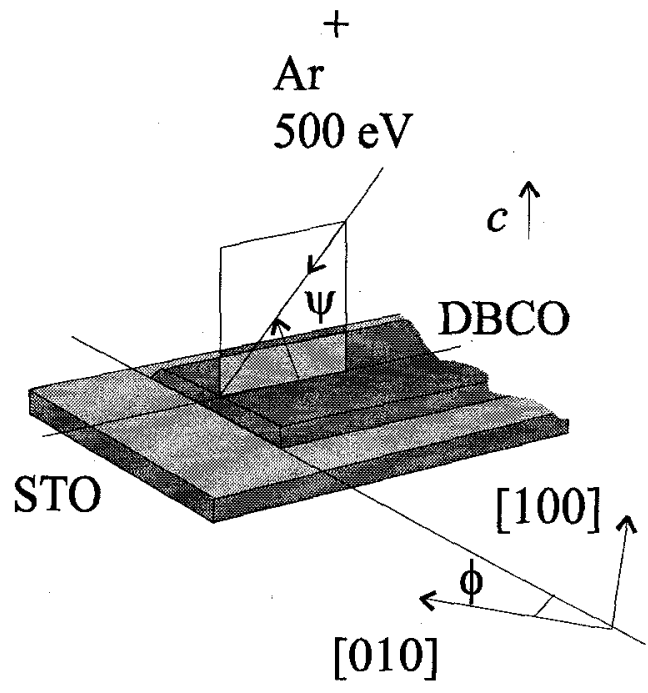

Figure 1: Schematic view of the geometry during etching. 
For morphology studies on ramps an "open air" Nanoscope III was used in AFM contact mode. with commercial $\mathrm{Si}_{3} \mathrm{~N}_{4}$ tips. Besides height data, also deflection data is collected. This data is essentially the derivative of the height, using high feedback gains, and is commonly referred to as the error-signal mode. This mode provides a sensitive edge detection technique.

\section{RESUlTS AND DISCUSSION}

The results of earlier experiments on the growth of barriers on etched ramped surfaces lead to the following discussion. After structuring the ramp, using a mask of photoresist, a curved, mostly concave, curvature occurs. In figure 2(a) and 2(b) AFM micrographs of a ramp made in a sputter deposited DyBCO film are given. The (photoresist) mask is aligned $\left(\phi \approx 0^{\circ}\right)$ with respect to one of the crystal axes of the $\mathrm{SrTiO}_{3}$ substrate. Figure 2(b) shows an enlargement of the ramp surface. Subtracting the average plane, the concave appearance of the ramp can be seen. This curvature of the ramp, due to the shift of the photoresist edge during etching, is also observed in HREM studies [3]. Besides this curvature, facets are observed, which are formed parallel to the ramp. These facets are caused by continuous surface reconstruction of the ramp during etching. The facets are about $20 \mathrm{~nm}$ wide and up to $500 \mathrm{~nm}$ long [4]. Misaligned ramps $\left(\phi \neq 0^{\circ}\right)$ show a less regular surface. Ramps, fabricated with photoresist masks, in films made by PLD, which have grain sizes much smaller than sputter deposited films, do not show these facets.

A barrier grown on top of the ramped surface showed, identical to misaligned ramps in sputtered films, large

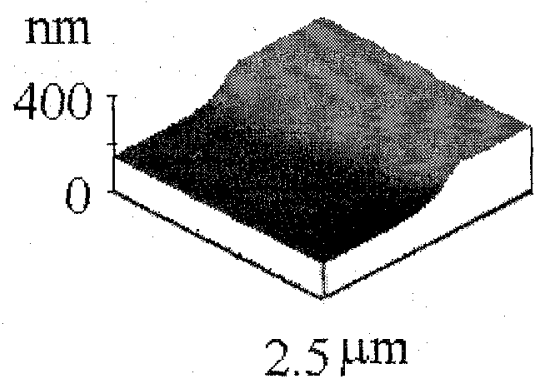

$\mathbf{a}$

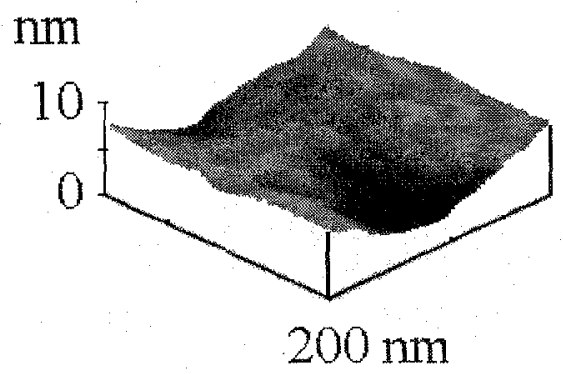

b

Figure 2: AFM micrographs of a ramp, made in sputter deposited DyBCO (a) and enlargement of the ramp surface with the average plane subtracted (b).
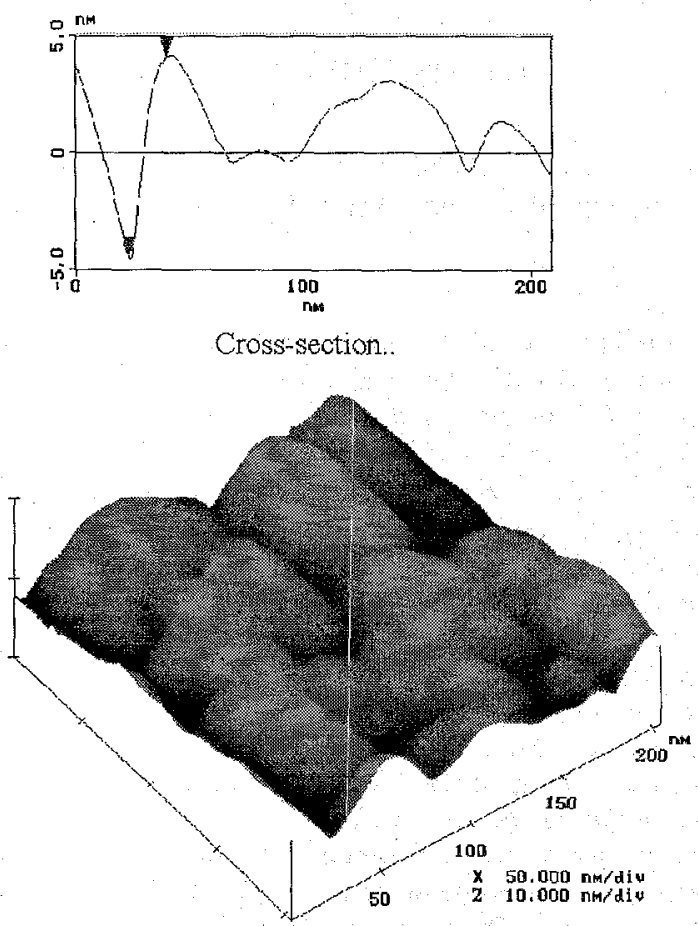

Figure 3: AFM micrograph and cross-section of a $6 \mathrm{~nm}$ barrier grown on a ramp, etched in a PLD film

boulders. In figure 3 an AFM micrograph and cross-section are given of a $6 \mathrm{~mm}$ thin barrier grown on a ramp; which is etched in a PLD film.

No long facets has been observed, due to the significantly smaller grain size of PLD films $(50-200 \mathrm{~nm})$ compare to sputter films $(\sim 1 \mu \mathrm{m})$. The height variations of the barrier can be twice as high than the overall deposited barrier thickness. These boulders will lead to undefined barriers and in the case of very small thickness $(<6 \mathrm{~nm})$, shorts will dominate.

To overcome this, we investigated if the ramp surface morphology could be altered, preferably leading to steps and facets of only a few unit-cells. Different samples were prepared by applying a photo resist mask with a grating image aligned with the crystal axis of the YBCO $(\phi \approx 0$ in fig. 1$)$. The ramps were etched with Ar-ion beam voltage of $500 \mathrm{~V}$ at an angle $\beta$ of 45 degrees. After etching of the ramp and the removal of photo resist, it was cleaned by another etching step with a beam voltage of $50 \mathrm{~V}$. This cleaning step improves the interface quality of the ramp significantly, as was confirmed by making ramp type junctions without a barrier. In this configuration, suppression of the critical current density is caused by the interface resistance, and, therefore, a measure for the total amount of damage that is caused during etching [5].

Hence, we tried to anneal a freshly etched ramp, at a temperature and oxygen pressure below the stability line in the phase diagram of YBCO [6]. It is believed that the surface layer is disordered due to ion beam etching and crystallization of this disordered YBCO might occur, leading to steps of only one unitcell height. 


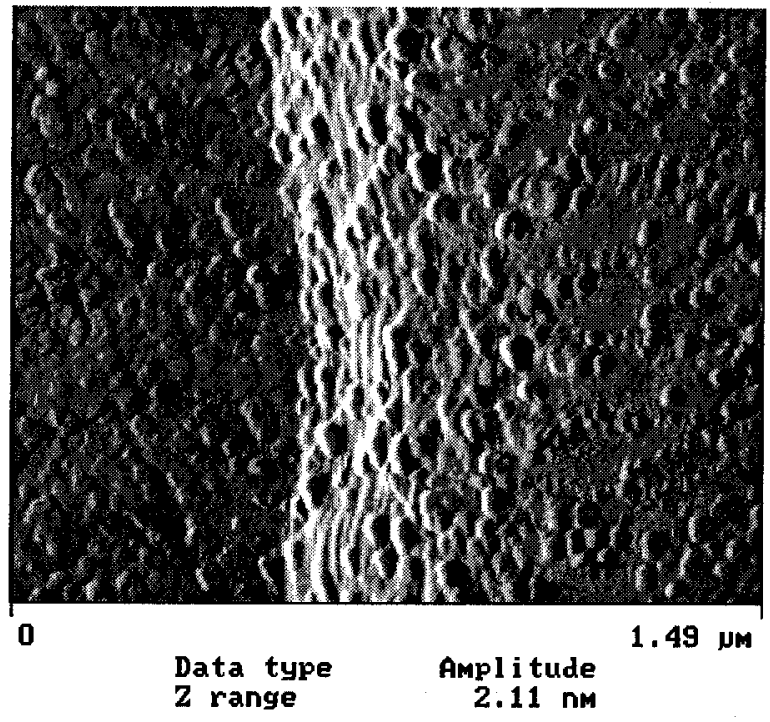

Figure 4: Deflection AFM micrograph of an annealed ramp surface

Subsequently, sample's ware annealed in 0.1 mbar oxygen for 1 hour at $800{ }^{\circ} \mathrm{C}$, and cooled in $200 \mathrm{mbar}$ oxygen to room temperature. The first pictures showed resemblance with earlier pictures: long facets and steps of several unitcells. In more detail, small structures are visible on top of the whole film, leading to an overall increase of roughness. In figure 4 the deflection data (derivative of the height or error-signal mode) of an AFM image is given. The deflection data provides a sensitive edge detection technique. Typical sizes of these features are about $20-40 \mathrm{~nm}$ in width and about 10 $\mathrm{nm}$ high. Detailed AFM studies showed that these structures were already present direct after the cleaning procedure with the Kaufman source. These structures were independent on the acceleration voltage or etch angle.

As a consequence, the quality of the ramped surface is predominantly determined by the etch rate of the mask, see Fig. 5. As the thickness of the etch mask decreases during etching, the shadow of the ion beam moves aside, resulting in a smaller angle in the ramp $(\alpha)$ than the etch angle $(\beta)$ and, by that, to an effective etch angle $(\gamma)$.

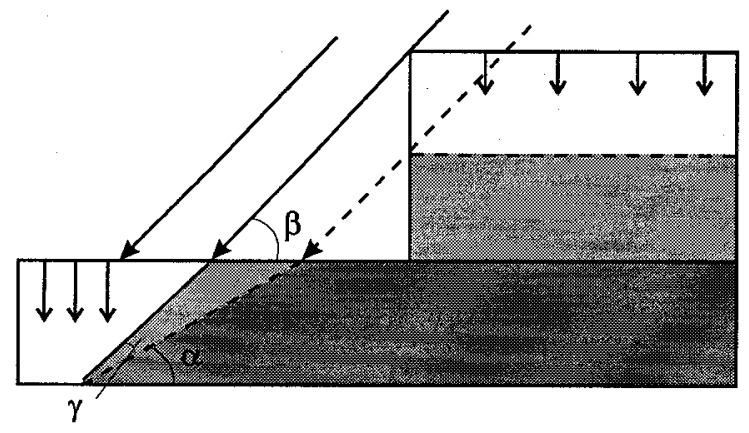

Figure 5: Influence of the etch rate of the mask to the ramp angle

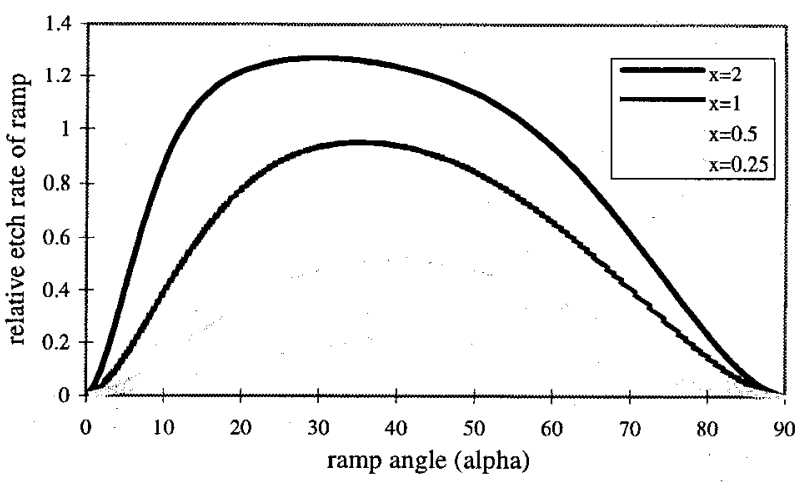

Figure 6: Relative etch rate on the ramp as a function of obtained ramp angle for different ratios in etch rate, $x=$ etch rate mask/etch rate film.

Fig. 6 shows the relative etch rate on the ramp as a function of obtained ramp angle for different ratios in etch rate, $x=$ etch rate mask/etch rate film. In this figure the angle dependent etch rate of $\mathrm{RBCO}$ is taken into account.

As an example we consider photoresist, which has an etch rate two times as large as RBCO $(x=2)$. As is shown in this graph, the effective angle $(\gamma)$ becomes very large (20-30 degrees) in the region of interest, usually an angle between 10 and 30 degrees. Taking into consideration the angular dependence of the etch rate, these angles lead to a significant etch rate on the ramp.

As can be seen from Fig. 6, the etch rate of the ramp area, and thus the surface damage can be suppressed by making use of harder etch masks, leading to a smaller etch rate on the ramp surface, which may lead to better surface qualities and a better template layer for the growth of barrier material.

As an alternative etch mask, we used titanium nitride (TiN). TiN has an etch rate, which is three times less then

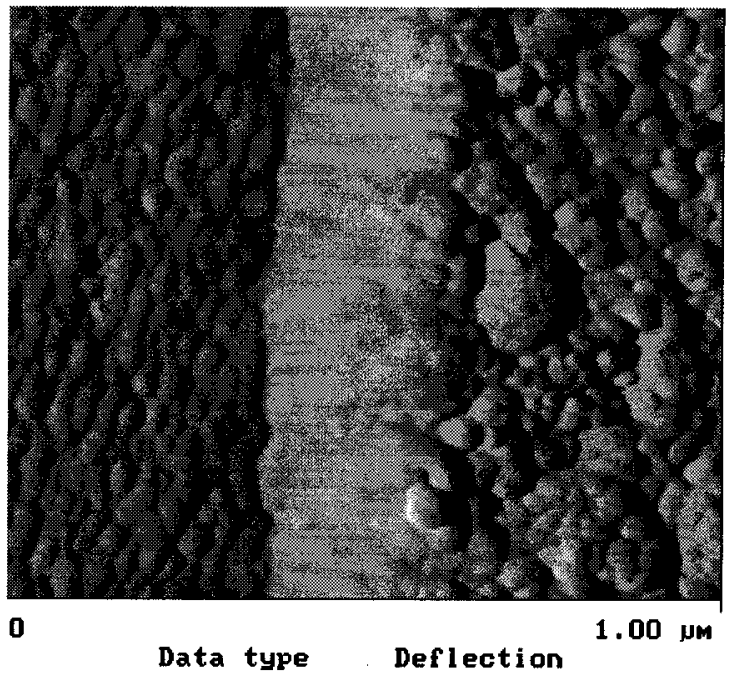

Figure 7: Deflection of the ramp area using hard TiN etch mask 


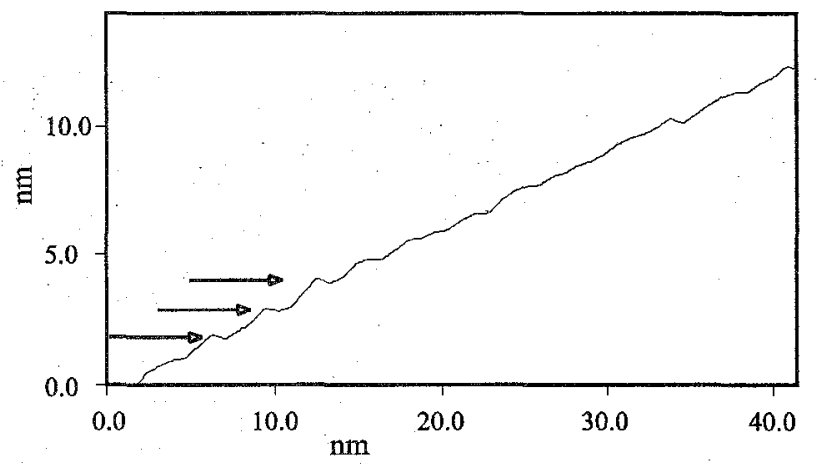

Figure 8: Cross section of zoom on annealed ramp. Arrows indicate steps with unitcell height.

RBCO. The quality of the ramp by means of homogeneity of the ramp angle and surface roughness was even better than expected. The ramp is very well defined: there is no concavity visible in the lower region of the ramp. The ramp area is completely smooth, and even when we zoomed, no structures were visible, as can be seen in Fig. 7 (note that the deflection mode is used to provide more sensitive height variations).

In addition, annealing experiments were performed on these ramps to see the influence of surface crystallization. This was done by annealing the sample slightly below the decomposition line in the phase diagram $(0.1$ mbar oxygen pressure, $780{ }^{\circ} \mathrm{C}$, for 1 hour, subsequently cooled to $450{ }^{\circ} \mathrm{C}$ and then cooled to room temperature in 200 mbar oxygen pressure). In Fig. 8, the cross-section of a zoom on an annealed ramp is given. The line scan shows steps of unitcell height on almost the whole ramp area. The steps of unitcell height indicate that the crystalline structure extends all the way up to the ramp surface, and that there is no 'damage region' present. In our search for a good barrier growth layer, a ramp surface of unit cell steps, as observed here, is needed if a step flow growth is desired.

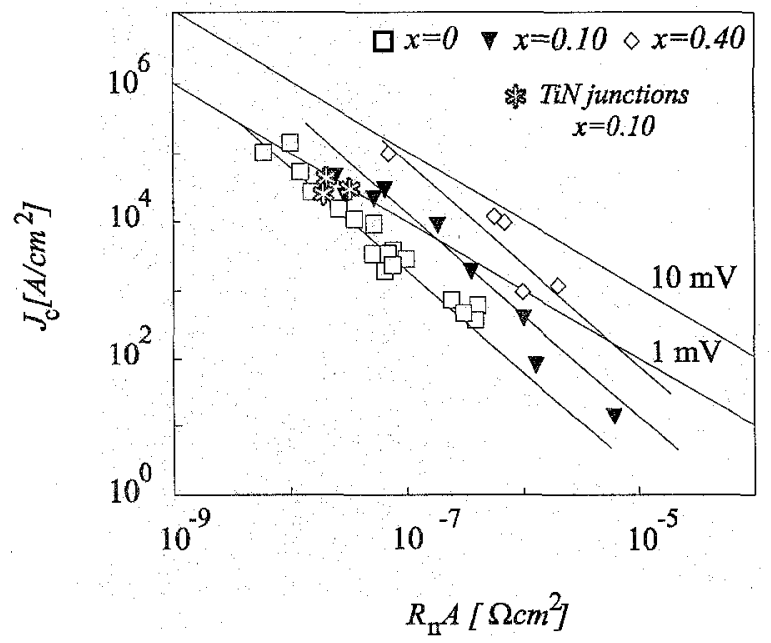

Figure 7: $J_{c}$ vs. $R_{n} A$ for junctions with $\operatorname{PrBa}_{2} \mathrm{Cu}_{3-x} \mathrm{Ga}_{x} \mathrm{O}_{7-\delta}$, barrier for different doping levels (from [7]), the stars indicate the junctions made with the TiN etch mask method (barrier doping $x=0.1$ )
With this technique, a first attempt has been made to fabricate Josephson junctions, using an in-situ technique. The thickness of the TiN mask was chosen such that it was completely removed after structuring the ramp. Direct after etching the ramp, the barrier and top electrode are deposited, without exhibition to air.

The junctions, with $10 \%$ Ga-doped PrBCO and thickness of $10 \mathrm{~nm}$, had $\mathrm{I}_{\mathrm{c}} \mathrm{R}_{\mathrm{n}}$ products of $0.8 \mathrm{mV}$ at $7 \mathrm{~K}$. The IV-curves all show RCSJ behaviour, Verhoeven published a systematic study with combinations of critical current densities and $R_{n} A$ products [7]. The junctions obtained in this work scale very well with the presented data, although, there is a tendency that the in-situ junctions have a somewhat lower $R_{n} A$ product. This property underwrites the suggestion of a smaller barrier resistance.

\section{CONCLUSIONS}

In this contribution we studied the effect of the masks used in multilayer ramp-structures. Using photoresist, a concave shape of the ramp area is observed. The area consists of facets and irregularities, even after etching at ambient temperature. Annealing of these structures will increase these anomalies. This damaged ramp surface is caused by the preferential sputter etching, due to a large angle between the angle of incidence and the ramp angle. The use of hard masks prevents this difference in angles. Furthermore, the effective etch rate for small angles minimizes this damaging effect. Steps of unit cell height are observed after annealing ramps fabricated using hard masks.

\section{REFERENCES}

[1] J. Gao, W.A.M. Aarnink, G.J. Gerritsma and H.Rogalla, Physica C 171; $126(1990)$.

[2] M.A.J. Verhoeven, A.A. Golubov, G.J. Gerritsma and H. Rogalla, IEEE Trans. Appl. Superc. 5, 2095 (1995).

[3] K. Verbist, O.I. Lebedev, G. Van Tendeloo, M.A.J. Verhoeven, A.J.H.M. Rijnders, Dave H.A. Blank, Low or high angle Ar ion-beam etching to create ramp-type Josephson Junctions?, to be published.

[4] M.A.J. Verhoeven, R. Moerman, M.E. Bijlsma, A.J.H.M. Rijnders, D.H.A. Blank, G.J. Gerritsma and H. Rogalla, Appl. Phys. Lett 68, 1276, (1996).

[5] M.A.J. Verhoeven, G.J. Gerritsma and H. Rogalla, Inst. Phys. Conf. EUCAS Ser. No. 148, 1395 (1995).

[6] R.H. Hammond and R. Bormann, Physica C 162-164, 703 (1989).

[7] M.A.J. Verhoeven, , G.J. Gerritsma, H. Rogalla and A.A. Golubov, App. Phys. Lett. 69, 848 (1996) 